\title{
Mild Ptosis Correction with the Stitch Method During Incisional Double Fold Formation
}

\author{
Edward Ilho Lee ${ }^{1}$, Tae Joo Ahn ${ }^{2}$ \\ ${ }^{1}$ Department of Plastic Surgery, Baylor College of Medicine, Houston, TX, USA; ${ }^{2}$ Gyalumhan Plastic Surgery, Seoul, Korea
}

Background Numerous methods exist for simultaneous correction of mild blepharoptosis during double eyelid surgery. These methods are generally categorized into either incisional (open) or non-incisional (suture) methods. The incisional method is commonly used for the creation of the double eyelid crease in patients with excessive or thick skin. However, concurrent open ptosis correction is often marred by the lengthy period of intraoperative adjustment, causing more swelling, a longer recovery time, and an increased risk of postoperative complications.

Methods The authors have devised a new, minimally invasive technique to alleviate mild ptosis during incisional double eyelid surgery. The anterior lamella is approached through the incisional technique for the creation of a double eyelid while the posterior lamella, including Muller's and levator muscles, is approached with the suture method for Muller's plication and ptosis correction.

Results The procedure described was utilized in 28 patients from June 2012 to August 2012. Postoperative asymmetry was noted in one patient who had severe preoperative conjunctival scarring. Otherwise, ptosis was corrected as planned in the rest of the cases and all of the patients were satisfied with their postoperative appearance and experienced no complications. Conclusions Our hybrid technique combines the benefits of both the incisional and suture methods, allowing for a predictable and easily reproducible correction of blepharoptosis with an aesthetically pleasing double eyelid.

Keywords Blepharoptosis / Muscles / Conjunctiva
Correspondence: Tae-Joo Ahn Gyalumhan Plastic Surgery, 21th, Mijinplaza, 390 Gangnam-daero, Gangnam-gu, Seoul 135-934, Korea Tel: $+82-2-535-6688$ Fax: +82-2-535-8580 E-mail: cmcanti@hanmail.net

No potential conflict of interest relevant to this article was reported.

Received: 2 Apr 2013 • Revised: 29 May 2013 • Accepted: 7 Jun 2013

pISSN: 2234-6163 • elSSN: 2234-6171 • http://dx.doi.org/10.5999/aps.2014.41.1.71 • Arch Plast Surg 2014;41:71-76

\section{INTRODUCTION}

Double eyelid surgery is now the most common cosmetic surgical procedure performed amongst East Asians and is synonymous with blepharoplasty in this patient population [1]. East Asians, in contrast to their Caucasian counterparts, typically have smaller eyes that often lack supratarsal creases, also known as double eyelid folds. Moreover, the lack of a supratarsal crease that causes hooding of the upper eyelid and the relative weak- ness of the upper eyelid elevators that reduces the vertical palpebral fissure height both produce the illusion of smaller eyes in Asians. By creating a supratarsal crease, either by an incisional or non-incisional method, the hooding of the skin is corrected by increasing the surface area of the skin as the skin invaginates inward. In addition, previously unrecognized blepharoptosis is frequently revealed in patients presenting for double eyelid surgery. Moreover, the creation of a supratarsal crease can unmask latent mild blepharoptosis or create it through iatrogenic injury

Copyright ( $\odot 2014$ The Korean Society of Plastic and Reconstructive Surgeons

This is an Open Access article distributed under the terms of the Creative Commons Attribution Non-Commercial License (http://creativecommons.org/

licenses/by-nc/3.0/) which permits unrestricted non-commercial use, distribution, and reproduction in any medium, provided the original work is properly cited.

www.e-aps.org 
to the levator complex. Therefore, concurrent ptosis correction is often needed, either through an incisional or a non-incisional method.

Several methods for non-incisional ptosis correction have been published. Shimizu et al. [2] utilized two to four separate threads to narrow the gap between the tarsus and levator muscle, which, in effect, plicates the Muller's muscle for ptosis correction. Around the same time, we introduced our method of nonincisional ptosis correction by tucking Muller's muscle through the triangular stitch method [3]. This method is composed of a single, running suture that locks soft tissue between the conjunctiva and Muller's muscle in a triangular configuration, which allows the thread to work as a loop or a sling in pulling up as much soft tissue as possible to correct ptosis. We, along with many others, believe that a single, running suture is preferable over multiple knots because it is able to distribute tension between thread points better, thus reducing the risk of suture loosening and allowing for more predictable results [2]. In cases of asymmetric ptosis correction, if there is a $1 \mathrm{~mm}$ difference in the eye opening strength between the eyes, then 2-loops are used for the weaker eye and 1-loop for the stronger eye to take into account the effect of Herring's law. If there is a $2 \mathrm{~mm}$ difference, then 2-loops are used for the weaker eye only.

The advantages of our technique include minimal to no scarring, quick recovery time, and minimal trauma to the eyelid tissue. Moreover, this technique is predictable and easily reproducible with low complication rates. Unfortunately, this technique should not be used in patients with loose or excess upper eyelid skin requiring skin excision or in patients with thick skin where the placement and effectiveness of the suture is unpredictable. In order to overcome limitations of the suture method in these patients, we have devised a hybrid technique whereby a double eyelid fold is created through an incisional method but ptosis is corrected through a non-incisional (suture) method.

\section{METHODS}

\section{Patient selection}

There are two steps to our method for patient selection. First, we determine whether the patient is a candidate for double eyelid surgery. If so, then we examine the upper eyelid skin for thickness and elasticity to determine whether the suture method is suitable. When patients present with excess or thick upper eyelid skin, the efficacy of the suture method is limited. Therefore, these types of patients require incisional double eyelid surgery.

Second, we determine whether the patient needs ptosis correction. We use marginal reflex distance 1 (MRD1) as a proxy to determine whether the patient has ptosis. Those with a MRD1 of 1 to $3 \mathrm{~mm}$, which we define as mild ptosis, are favorable candidates for correction with our suture method. Notably, we do not recommend the suture method for patients with severe ptosis, as they will require a more extensive procedure to achieve desirable correction.

Once it is determined that the patient needs an open approach for the skin and has mild ptosis, the following steps are followed.

\section{Surgical technique}

With the patient in a seated position, a line for the location of the double eyelid crease is identified and marked. Next, the amount of excess upper eyelid skin is determined and the area of skin excision is marked. At this point, the degree of ptosis on the two sides is compared, and the amount of ptosis correction needed is established. The patient is then brought to the operating room, where she is laid in a supine position. All procedures are performed under conscious sedation and local anesthetics. After confirming all markings and injecting local anesthetic, an incision is made along the line demarcating the excess skin excision. The incision is carried down to the orbital septum and the excess skin and orbicularis muscle is excised. If necessary, the orbital septum is accessed to tease out and excise any excess orbital fat. Then, ptosis correction is performed with either the 1-loop or the 2-loop suture technique as described below.

\section{1-Loop technique}

First, three points are marked on the conjoint tendon perpendicular to the upper margin of the tarsal plate as follows: 1) medial limbus; 2) midpupillary line; and 3) lateral limbus (Fig. 1). A 7-0 nylon suture on a 24-mm long needle is inserted from directly above the upper margin of the tarsal plate and passed through point 3 and out through the conjunctiva. Next, Muller's muscle tucking is performed by passing this suture approximately 8 to $10 \mathrm{~mm}$ cephalad and then passing it back caudally to the upper border of the tarsal plate. At this point the upper border of the tarsal plate should be directly above the midpupillary line. The suture is next passed anteriorly from the upper border of the tarsal plate through point 2 , then towards point 3 while securing a portion of the conjoint tendon and finally tied there.

\section{2-Loop technique}

The steps as described above are followed up to the completion of the first Muller's tucking procedure. After passing the suture anteriorly from the upper border of the tarsal plate through point 2 , the suture is routed towards point 1 while securing a portion of the conjoint tendon. At point 1 , the suture is inserted through the upper margin of the tarsal plate and passed out through the conjunctiva directly below the point. Next, Muller's muscle 


\section{Fig. 1. Schematic diagram of 2-loop suture}

(A) Schematic diagram of the 2-loop suture technique to tuck Muller's muscle for ptosis correction. Design of the 2-loop technique during incisional double eyelid surgery, as viewed from (B) the anterior lamella and from (C) conjunctiva.

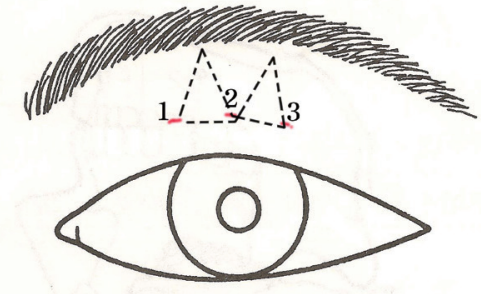

(A)
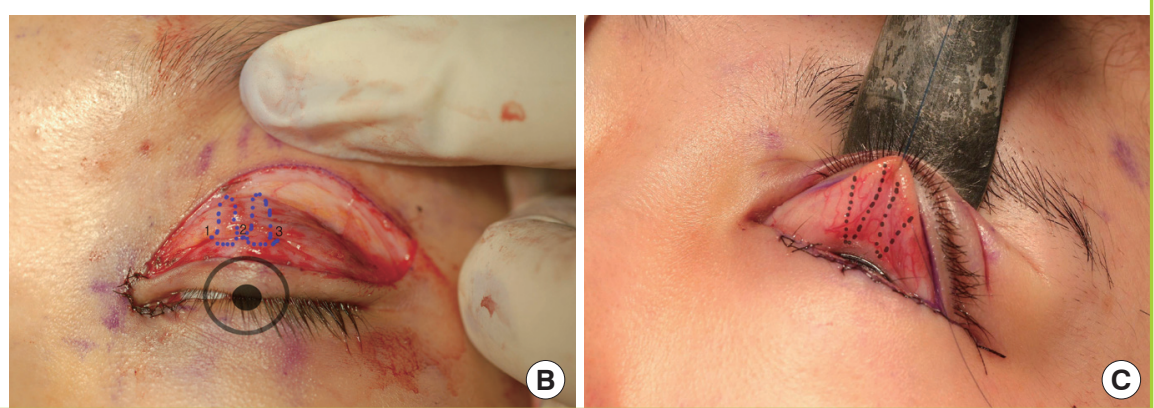

\section{Fig. 2. Case 1: preoperative and postoperative appearance}

A 19-year-old female. Incisional double eyelid surgery combined with the 2-loop suture technique for ptosis correction. (A) Preoperative appearance with $2 \mathrm{~mm}$ marginal reflex distance 1 (MRD1) on both eyes, which appeared to be worse due to hooding of excess skin causing effacement of the lid margin. (B) Appearance 3 months postoperatively with an MRD1 of $5 \mathrm{~mm}$ on both eyes. (C) Intraoperative view of the conjunctiva after completion of the 2-loop suture technique to tuck Muller's muscle.
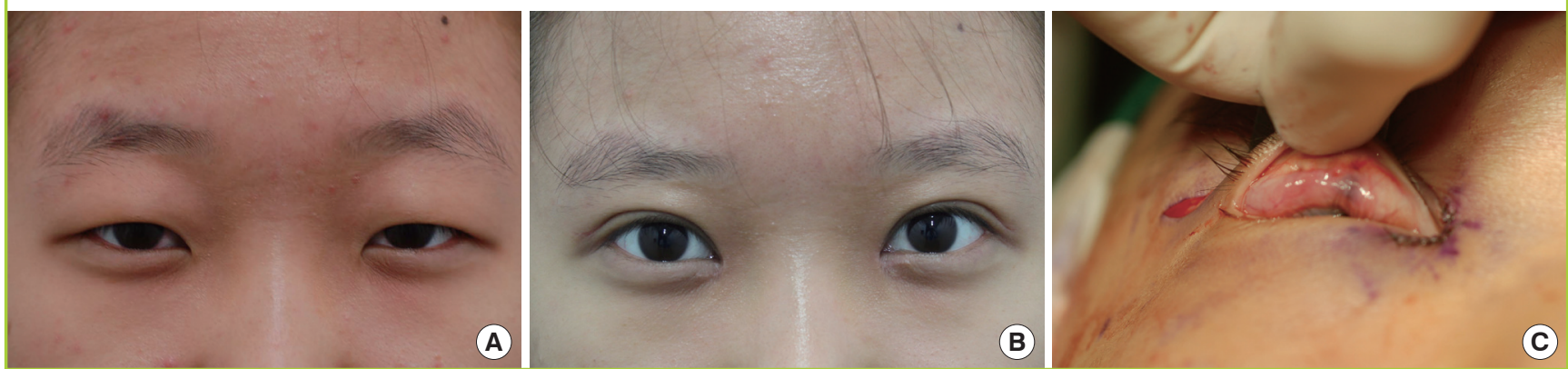

tucking is performed again and the needle is passed through point 2 . The suture is then passed toward point 3 while securing a portion of the conjoint tendon and then tied here.

For both the 1-loop and 2-loop techniques, the protrusion in the conjunctiva and Muller's muscle evens out and flattens over time so that it does not irritate the eye. After completion of ptosis correction, the supratarsal fold is created and the incisional double eyelid surgery is completed in a standard manner.

\section{RESULTS}

The procedure described was utilized in 28 patients from June 2012 to August 2012. Postoperative asymmetry was noted in one patient who had severe preoperative conjunctival scarring. Otherwise, no other complications were noted over a minimum follow-up period of 3 months.

\section{Case 1}

A 19-year-old female presented with $2 \mathrm{~mm}$ MRD1 on both eyes, which appeared to be worse due to hooding of excess skin causing effacement of the lid margin. Excess skin and muscle were excised through the incisional method, which also resulted in adhesion-like septated tissue around the septum being freed up. The MRD1 measurements at 3 months postoperatively were 5 mm on both eyes (Fig. 2).

\section{Case 2}

A 28-year-old female presented complaining of sunken eyes and discomfort when opening her eyes. The skin and muscle excision was kept at a minimum and the 2-loop technique as described above was used for ptosis correction. MRD1 was increased from $3.5 \mathrm{~mm}$ preoperatively to $5.5 \mathrm{~mm}$ at 8 months postoperatively. Resolution of discomfort with eye opening and the correction of sunken eyes was maintained at 8 months postoperatively (Fig. 3).

\section{Case 3}

A 31-year-old female, with a history of double eyelid surgery 4 years earlier through a partial incision technique, presented complaining that her eyes still appeared small and tired. The double eyelid was revised and ptosis was corrected with our technique as described above. The MRD1 was increased from $2.5 \mathrm{~mm}$ preop- 


\section{Fig. 3. Case 2: preoperative and postoperative appearance}

A 28-year-old female. Incisional double eyelid surgery combined with the 2-loop suture technique for ptosis correction. (A,B) Preoperative appearance with sunken eyes and a $3.5 \mathrm{~mm}$ marginal reflex distance 1 (MRD1) on both eyes. (C) Appearance 8 months postoperatively with an MRD1 of $5.5 \mathrm{~mm}$ on both eyes.
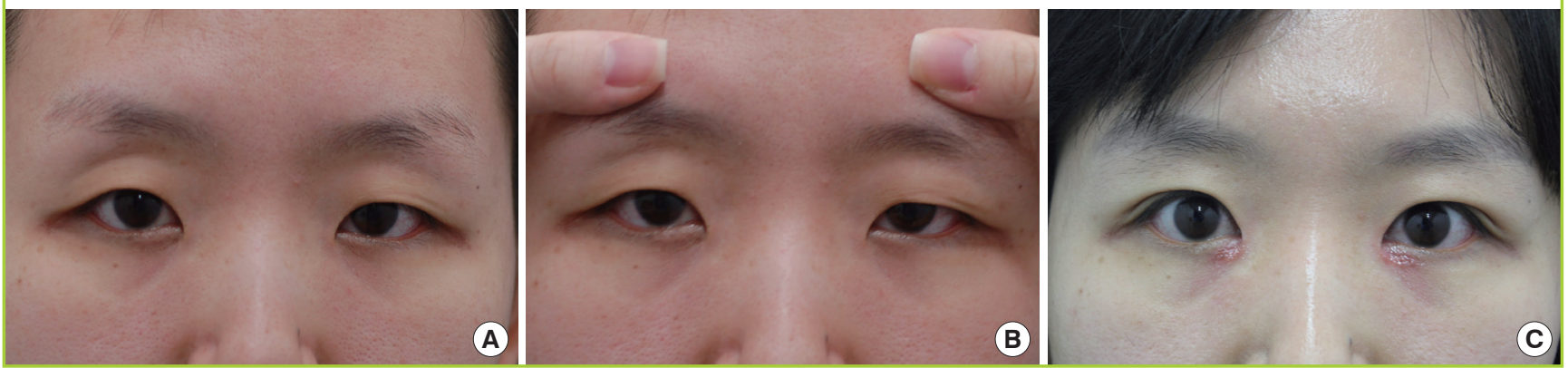

Fig. 4. Case 3: preoperative and postoperative appearance

A 31-year-old female. Incisional double eyelid surgery combined with the 2-loop suture technique for ptosis correction. (A) Preoperative appearance with a history of double eyelid surgery 4 years earlier through the partial incision technique, and a complaint of small and tired eyes. (B) Appearance 3 months postoperatively after double eyelid revision and ptosis correction. Marginal reflex distance 1 was increased from $2.5 \mathrm{~mm}$ preoperatively to $5 \mathrm{~mm}$.
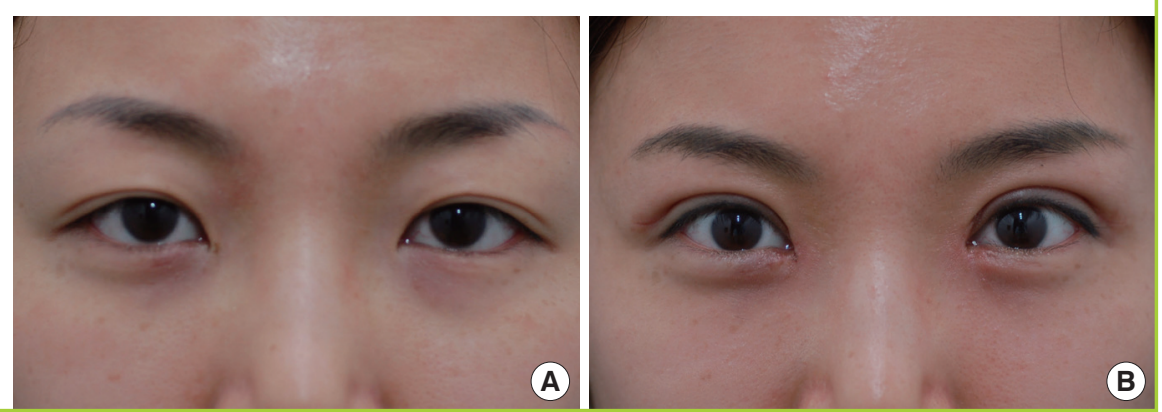

Fig. 5. Case 4: preoperative and postoperative appearance

A 46-year-old female. Incisional double eyelid surgery combined with a 1-loop (right) and 2-loop (left) suture technique for ptosis correction. (A) Preoperative appearance. The marginal reflex distance 1 (MRD1) on the left was $0.5 \mathrm{~mm}$ smaller. (B) Preoperatively, the asymmetry was more pronounced when frontalis muscle action was blocked, with an MRD1 difference of $1 \mathrm{~mm}$. (C) Appearance 3 months postoperatively with an MRD1 of $3 \mathrm{~mm}$ on both eyes.

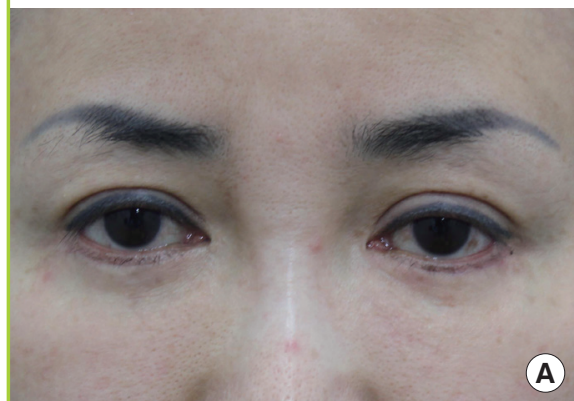

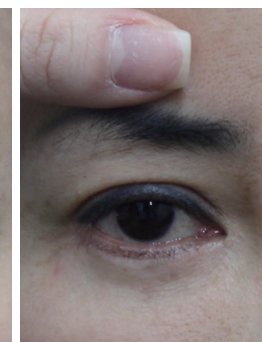

(A)

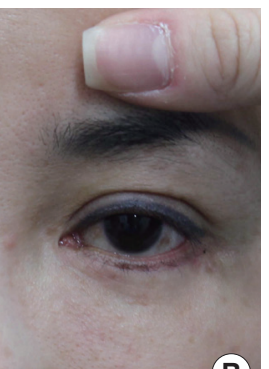

(B)

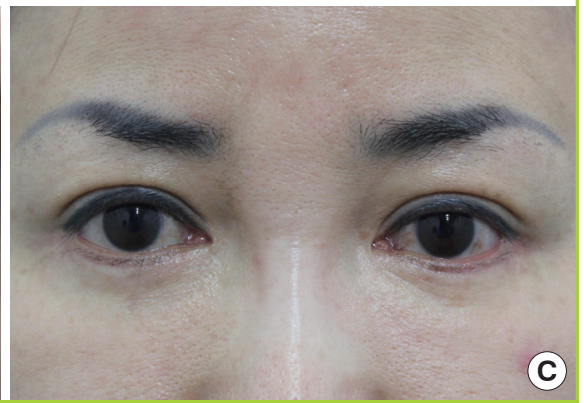

eratively to $5 \mathrm{~mm}$ at 3 months postoperatively (Fig. 4).

\section{Case 4}

A 46-year-old female, with a history of bilateral upper and lower blepharoplasty 10 years earlier, presented for revision. The MRD1 on the right was $2 \mathrm{~mm}$ and on the left was $1.5 \mathrm{~mm}$. The asymmetry became more pronounced when the frontalis muscle action was blocked, with an MRD1 of $2 \mathrm{~mm}$ on the right and $1 \mathrm{~mm}$ on the left. Incisional double eyelid surgery was performed on both eyes, with 1-loop on the right and 2-loops on the left for ptosis correction. At 3 months postoperatively, an MRD1 of $3 \mathrm{~mm}$ was maintained bilaterally with improvements in the lower scleral show phenomenon (Fig. 5).

\section{DISCUSSION}

The method for correcting blepharoptosis varies depending upon its severity and cause. Numerous operative methods have been reported; however, variables such as local anesthetics, hematoma, swelling, and elasticity of the muscle make verifying 


\section{Fig. 6. Cross-sectional view and intraoperative view}

Cross-sectional view of Muller's muscle tucking with the 2-loop technique.

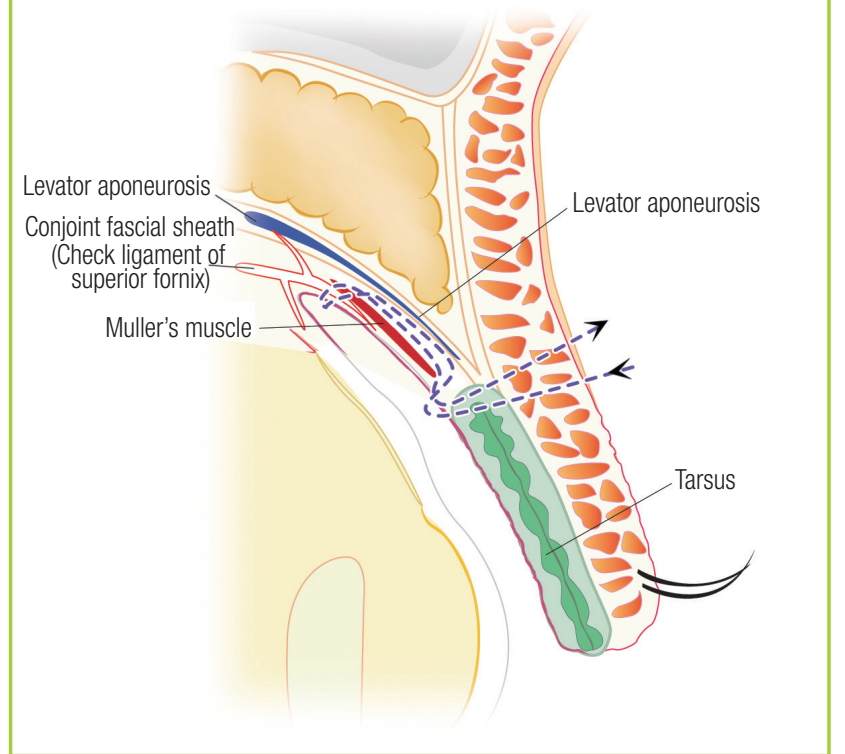

and adjusting the degree of ptosis correction challenging with these methods $[2,3]$.

Capitalizing on our experience with the non-incisional method for simultaneous double eyelid and ptosis correction, the current technique was crafted to: 1) allow the creation of a double eyelid fold in patients with loose or thick skin; 2) minimize the risk of complications in cases of mild ptosis by simplifying the procedure and maintaining integrity of the posterior lamella through the non-incisional method; and 3) minimize asymmetry in ptosis correction while eliminating the need for the patient to sit up during the procedure to check for symmetry.

Although an incision is not created in order to correct ptosis (the posterior lamella is not violated either by incision or detachment), we believe that Muller's plication technique creates scar casting by permanently shortening the muscle. In addition, by using the suture method for concomitant ptosis repair instead of the traditional open technique, we are able to preserve the integrity of both the Muller's and levator muscles. Moreover, the levator may appear to have had levator complex advancement because it is likely that the levator complex is being pulled with our technique (Figs. 1, 6).

According to Hirasawa et al. [4], the eye-elevating muscle is composed of voluntary fast-twitch muscle fibers and involuntary slow-twitch muscle fibers. The initial, voluntary, and quick muscle contraction leads to the stretching of the Muller's muscle. This contraction elicits an involuntary response from the muscles to strengthen the eye opening reflex.

To sum up, the effectiveness of our suture method for ptosis repair is based on the following: 1) the reflex action, as described above, that is reinforced with tucking of the Muller's muscle; 2) posterior lamella shortening; 3 ) creation of a sling for the conjoint fascial sheath; and 4) levator advancement.

Although our experience with the current method described (incisional method for double eyelid creation and suture method for ptosis correction) is limited, our experience with the nonincisional method for simultaneous double eyelid creation and mild ptosis correction is more extensive, and we decided to analyze these results in order to assess the utility of this technique. From January 2011 to December 2012, the non-incisional method for simultaneous double eyelid creation and mild ptosis correction was utilized in 371 patients with an average of 6 months of follow-up. In all of the patients, the number of loops to use, and therefore, the degree of ptosis correction, was decided preoperatively, which allowed judicious use of local anesthetics during the procedure for patient comfort, attributing any small degree of intraoperative asymmetry to the effect of local anesthetics. There were 7 revision cases, including 3 cases of suture failure, 2 of asymmetry, 1 unsatisfactory result due to what was perceived as an excessively deep supratarsal fold, and another unsatisfactory result due to lateral hooding created by eyebrow descent from improvement in eyelid ptosis. Revision was performed in one patient because she wanted smaller folds. The case of asymmetry was easily corrected by adding 1-loop to the more ptotic side under local anesthesia.

Since we have used this method, the utility, safety, and predictability of Muller tucking through the triangular stitch method (also known as a conjoint fascial sheath sling) was realized and applied to open double eyelid surgery to address patients with thick or excessive skin who were previously thought not to be ideal candidates for the non-incisional method for ptosis correction. Moreover, in the cases of incisional double eyelid surgery with symmetric mild ptosis, the 1-loop or 2-loop techniques were easily added without the need to sit patients up during surgery to confirm symmetric ptosis correction, thus reducing operative times.

When compared to the traditional open ptosis repair technique, our loop technique has the following advantages: 1) It can be performed as fast as 5 minutes per eyelid without the need to sit patients up intra-operatively to confirm ptosis correction, thus saving time; 2) although the anterior lamella is incised in both techniques, posterior lamella, which is commonly detached or incised in open ptosis repair, is preserved, thus reducing recovery time; and 3) ample amounts of local anesthetics can be administered since intra-operative adjustments are not needed, thus improving patient comfort during the procedure.

From our experience, $1 \mathrm{~mm}$ and $2 \mathrm{~mm}$ of ptosis correction can 
be achieved predictably using 1-loop and 2-loops, respectively, in patients with soft or thin eyelid skin. However, the degree of ptosis correction using the suture technique could be reduced by as much as $50 \%$ due to the texture of the thick, scarred skin in patients with previous trauma. In these patients, the skin's added thickness and weight, along with adhesions from previous trauma, likely contribute to this reduced effectiveness. Moreover, this technique is least successful in patients who have severe conjunctival scarring from strabismus surgery during childhood. Such cases highlight the potential for scars to hinder a surgery that attempts to augment levator function. The incisional method to approach the anterior lamella allows for lysis of adhesions and separation of scar tissue. This greatly improves the quality of the skin and compliments the suture method by allowing it to be more effective and predictable. In case 4 , the degree of lysis was minimized by going through previous blepharoplasty incision, and ptosis was predictably corrected with our stitch method by utilizing tracks from the previous suture method for double eyelid creation. In general, for patients with symmetric mild ptosis in the setting of thick or scarred skin, the 2-loop technique is recommended over the 1-loop technique due to its greater ability to overcome soft tissue limitations. We plan on publishing our long-term results of the suture method for ptosis correction with either the incisional or suture method for double eyelid surgery in the near future.

Technically, the location of the suture should be at a depth underneath the conjunctiva and Muller's muscle so that if the needle is lifted during Muller tucking of the posterior wall, the suture should be visible. Additionally, it is important to note that if the plication is performed at a deeper depth, its effect can take longer to become visible in the postoperative period. Therefore, although the results of this method are predictable, the eyes can appear to be deceptively asymmetric for two to three months depending on the depth of plication and the amount of postoperative swelling.

Our proposed suture technique further stabilizes the integrity and function of the stitch by placing the stitch between the upper margin of the tarsal plate and the conjoint fascial sheath [5] above Muller's muscle for the Muller's muscle plication, allowing for longer tails on the knot since the knot can be buried at this interface.

Furthermore, the volume of local anesthetics used is of minimal concern when performing our technique. Therefore, the pain management of patients occurs more seamlessly. With current techniques, local anesthetics are used cautiously because these techniques require intraoperative adjustments for ptosis correction and cannot account for post-operative asymmetry resulting from using a higher volume of local anesthetic. In contrast, since the degree of ptosis correction is solely dependent on the number of loops used, our technique can accommodate a more liberal usage of local anesthetic for the patient.

The non-incisional suture method technique has gained immense popularity among young Asian females who desire double eyelid creases $[6,7]$. In today's fast-paced society, a surgically effective method for correcting blepharoptosis is needed along with a minimal recovery period. Despite the need for skin incision for double eyelid creation, there is no additional incision or detachment of structures for blepharoptosis correction with our technique.

In conclusion, this article introduces a minimally invasive technique to conduct double eyelid creation simultaneously with mild blepharoptosis correction. The advantages of our new, hybrid technique are predictable results, minimal scarring, quick recovery time, and reduced trauma to the eyelid tissue. And finally, this method is technically simple and easily reproducible with a relatively short operative time.

\section{REFERENCES}

1. Li J, Lin M, Zhou H, et al. Double-eyelid blepharoplasty incorporating blepharoptosis surgery for 'latent' aponeurotic ptosis. J Plast Reconstr Aesthet Surg 2011;64:993-9.

2. Shimizu Y, Nagasao T, Asou T. A new non-incisional correction method for blepharoptosis. J Plast Reconstr Aesthet Surg 2010;63:2004-12.

3. Ahn TJ. Blepharoptosis correction with stitch method. J Korean Soc Aesthetic Plast Surg 2010;16:167-70.

4. Hirasawa C, Matsuo K, Kikuchi N, et al. Upgaze eyelid position allows differentiation between congenital and aponeurotic blepharoptosis according to the neurophysiology of eyelid retraction. Ann Plast Surg 2006;57:529-34.

5. Hwang K, Shin YH, Kim DJ. Conjoint fascial sheath of the levator and superior rectus attached to the conjunctival fornix. J Craniofac Surg 2008; 19:241-5.

6. Ryu HS, Kum IS, Minn KW. Cosmetic double eyelid surgery: single $5 \mathrm{~mm}$-partial incision method. J Korean Soc Plast Reconstr Surg 2002;29:521-5.

7. Kim YK, Kwon JD, Oh KS. Double eyelid operation with three tiny incisions. J Korean Soc Plast Reconstr Surg 2000; 27:195-8. 